\title{
Inespecificidade parasitária em Argas (Persicargas) miniatus Koch, 1844 (Acari: Argasidae)
}

[Nonspecificity of parasitism on Argas (Persicargas) miniatus Koch, 1844 (Acari: Argasidae)]

\author{
E.S. Lorosa ${ }^{1}$, R.E. Andrade ${ }^{1}$, M.V.M. Valente ${ }^{1}$, M.S.Faria ${ }^{1}$, J.R. Cruz ${ }^{1}$, G.S. Gazeta ${ }^{1,2 *}$. \\ ${ }^{1}$ Instituto Oswaldo Cruz - Fiocruz \\ Av. Brasil, 4365 - Manguinhos \\ 21045-900 - Rio de Janeiro, RJ. \\ ${ }^{2}$ Bolsista CNPq.
}

\begin{abstract}
RESUMO
Foram coletados 665 espécimes de Argas miniatus em dois municípios dos estados da Bahia e Minas Gerais. Destes, 596 (89,6\%) tinham se alimentado, sendo que 489 (82\%) reagiram para um único tipo de sangue, distribuídos entre aves $(46,8 \%)$, roedores $(30,9 \%)$, gambás $(14,5 \%)$, bovinos $(4,3 \%)$ e eqüinos (3,5\%). Nesse tipo de reação, o sangue de mamíferos foi detectado em 53,2\% (260/489) dos argasídios. As reações múltiplas foram observadas em 107 (17,9\%) carrapatos, com sangue de aves presente em $84,1 \%$ (90/107), enquanto, o sangue de mamíferos reagiu em 100\% (107/107). Os resultados apontam para a inespecificidade parasitária.
\end{abstract}

Palavras-chave: carrapato, Argas, especificidade, precipitina

\begin{abstract}
Six hundred and sixty-five specimens of Argas miniatus were collected in two municipalities of Bahia and Minas Gerais states, Brazil. Five hundred and ninety-six (89.6\%) of them had fed and 489 (82\%) of them reacted to only one type of blood, including birds (46.8\%), rodents (30.9\%), opossuns (14.5\%), bovines (4.3\%) and horses (3.5\%). In that reaction, the type of mammal blood was detected in 53.2\% (260/489) of the ticks. Multiple reactions were observed in 107 (17.9\%) ticks, with blood of birds present in 84.1\% (90/107), while the blood of mammals was detected in 100\% (107/107). The results point for the nonspecificity of parasitism.
\end{abstract}

Keywords: tick, Argas, host specificity, precipitin

\section{INTRODUÇÃO}

Argas (Persicargas) miniatus Koch, 1844 (Acari: Argasidae) é uma espécie de carrapato de distribuição neotropical e neártica, que parasita aves em ambiente silvestre e antrópico (Flechtmann, 1973; Camicas et al., 1998, Evans et al., 2000; Serra-Freire, 2001). Clinicamente está envolvida em quadros de anemia profunda, reações alérgicas, toxicoses, espiroquetose, borreliose de aves e, eventualmente, óbito (Flechtmann, 1973; Serra-Freire, 2001).
Além de diferenças morfológicas, aspectos biológicos únicos dos argasídeos sugerem características particulares no processo de adaptação e especificidade ao hospedeiro e ambiente. Assim, larvas podem se fixar por poucos dias ao hospedeiro, mas ninfas e adultos nunca se alimentam por muito tempo. Igualmente, o número de estádios na fase de ninfa é único entre os carrapatos, bem como as características de oviposição. Dessa forma, apesar de a evolução dos carrapatos ter sido

Recebido em 19 de abril de 2006

Aceito em 17 de setembro de 2007

* Autor para correspondência (corresponding author)

E-mail: gsgazeta@ioc.fiocruz.br 
freqüentemente associada à especificidade com o hospedeiro, aceita-se a idéia que os parasitos são dependentes de seus hospedeiros (Hoogstraal e Aeschlimann, 1982; Hoogstraal e Kim, 1985). Klompen et al. (1996) chamam a tenção de que Argasidae está presente em ninhos, tocas e poleiros, usualmente freqüentados por um único hospedeiro ou por indivíduos da mesma espécie hospedeira, acasalando-se fora do corpo do hospedeiro. Este comportamento faria com que se alimente em um mesmo indivíduo ou grupo de indivíduos de uma mesma espécie, em todos os estádios, durante seu ciclo de vida.

O objetivo deste trabalho é analisar o potencial de inespecificidade parasitaria de $A$. miniatus.

\section{MATERIAL E MÉTODOS}

Durante atividade de captura de triatomíneos, realizada pela equipe do Laboratório Nacional e Internacional de Referência em Taxonomia de Triatomíneos - Fiocruz - RJ, foi observada a presença de carrapatos em diferentes ambientes examinados. A coleta manual dos carrapatos foi realizada no peridomicílio das localidades de Queimada Nova e Corguinho, município de Rafael Jambeiro, estado da Bahia (S $12^{\circ} 24.30 \mathrm{~W}$ $39^{\circ} 30.05$ ), com temperatura média de $24^{\circ} \mathrm{C}$ e altitude de $238 \mathrm{~m}$; e na localidade de Barra da Sepultura, município de Gouveia, estado de Minas Gerais (S 18 27.16 W 43044.27), com temperatura média de $19,4^{\circ} \mathrm{C}$ e altitude de $1.113 \mathrm{~m}$.

No município de Rafael Jambeiro os espécimes foram coletados no período de 5 a 15 de maio de 2004, em currais onde havia a presença de diferentes espécies de mamíferos. Em Gouveia, a coleta ocorreu de 7 a 13 de agosto de 2004, em galinheiro próximo a um chiqueiro. Os espécimes coletados foram acondicionados em frascos de polietileno, rotulados segundo a procedência e armazenados em caixa térmica, sendo transportados vivos ao laboratório de base, montado no campo, onde os carrapatos foram anestesiados pela exposição de vapores de clorofórmio e congelados a $-20^{\circ} \mathrm{C}$, para a interrupção do processo digestivo, até a realização do teste de precipitina.

Os espécimes foram encaminhados ao Laboratório de Ixodides, Departamento de Entomologia - Fiocruz, RJ, onde os adultos foram identificados, segundo chave dicotômica de Aragão e Fonseca (1961), e separados os estádios oriundos das diferentes localidades. As amostras, assim separadas, foram encaminhadas ao setor de precipitina do LNIRT, onde foram trituradas inteiras em tubo de ensaio com solução salina $(\mathrm{NaCl} 0,85 \%)$ e submetidas à técnica de precipitina (Siqueira, 1960), modificada por Lorosa et al. (1998). Foram utilizados anti-soros de ave, roedor, gambá, bovino, eqüino e suíno.

\section{RESULTADOS}

Foram coletados 665 carrapatos identificados como Argas (Persicargas) miniatus Koch, 1844, em diferentes estádios. Quinhentos e noventa e seis $(89,6 \%)$ haviam alimentado, sendo que, entre estes, $489(82 \%)$ reagiram para um único tipo de sangue hospedeiro e em 107 (17,9\%) dois ou três tipos de sangue foram detectados (Tab. 1).

Reações simples foram observadas em todos os estádios. Sangue de ave foi detectado em 229 $(46,8 \%)$ carrapatos, de roedor em 151 (30,9\%), de gambá em 71 (14,5\%), de bovino em 21 $(4,3 \%)$ e de eqüino em 17 (3,5\%) (Tab. 2). Nesse tipo de reação o sangue de mamíferos foi detectado em 53,2\% (260/489) dos argasídeos.

Tabela 1. Freqüências absoluta $(\mathrm{N})$ e relativa (\%) de reações do teste de precipitina, segundo o estádio de Argas (Persicargas) miniatus e o município pesquisado

\begin{tabular}{|c|c|c|c|c|c|c|c|c|c|c|c|c|c|c|c|c|c|c|}
\hline \multirow{3}{*}{ Reação } & \multicolumn{6}{|c|}{ Raphael Jambeiro - BA } & \multicolumn{4}{|c|}{ Gouveia - MG } & \multicolumn{6}{|c|}{ Total } & \multirow{2}{*}{\multicolumn{2}{|c|}{ Total geral }} \\
\hline & \multicolumn{2}{|c|}{ Larvas } & \multicolumn{2}{|c|}{ Ninfas } & \multicolumn{2}{|c|}{ Adultos } & \multicolumn{2}{|c|}{ Ninfas } & \multicolumn{2}{|c|}{ Adultos } & \multicolumn{2}{|c|}{ Larvas } & \multicolumn{2}{|c|}{ Ninfas } & \multicolumn{2}{|c|}{ Adultos } & & \\
\hline & $\mathrm{N}$ & $\%$ & $\mathrm{~N}$ & $\%$ & $\mathrm{~N}$ & $\%$ & $\mathrm{~N}$ & $\%$ & $\mathrm{~N}$ & $\%$ & $\mathrm{~N}$ & $\%$ & $\mathrm{~N}$ & $\%$ & $\mathrm{~N}$ & $\%$ & $\mathrm{~N}$ & $\%$ \\
\hline Simples & 28 & 68,29 & 79 & 84,94 & 290 & 72,50 & 34 & 72,34 & 58 & 69,05 & 28 & 68,29 & 113 & 80,71 & 348 & 71,90 & 489 & 73,53 \\
\hline Dupla/tripla & 0 & 0 & 5 & 5,38 & 88 & 22,00 & 4 & 8,51 & 10 & 11,90 & 0 & 0 & 9 & 6,43 & 98 & 20,25 & 107 & 16,09 \\
\hline Não reagiu & 13 & 31,71 & 9 & 9,68 & 22 & 5,50 & 9 & 19,15 & 16 & 19,05 & 13 & 31,71 & 18 & 12,86 & 38 & 7,85 & 69 & 10,38 \\
\hline Total & 41 & 100 & 93 & 66,43 & 400 & 82,64 & 47 & 33,57 & 84 & 17,36 & 41 & 6,17 & 140 & 21,05 & 484 & 72,78 & 665 & 100 \\
\hline
\end{tabular}


Inespecificidade parasitária...

Tabela 2. Freqüência absoluta $(\mathrm{N})$ e relativa (\%) de hospedeiros detectados em reações simples no teste de precipitina, segundo o estádio de Argas (Persicargas) miniatus e o município pesquisado

\begin{tabular}{|c|c|c|c|c|c|c|c|c|c|c|c|c|c|c|c|c|c|c|}
\hline \multirow{3}{*}{ Hospedeiro } & \multicolumn{6}{|c|}{ Raphael Jambeiro - BA } & \multicolumn{4}{|c|}{ Gouveia - MG } & \multicolumn{6}{|c|}{ Total } & \multirow{2}{*}{\multicolumn{2}{|c|}{ Total geral }} \\
\hline & \multicolumn{2}{|c|}{ Larvas } & \multicolumn{2}{|c|}{ Ninfas } & \multicolumn{2}{|c|}{ Adultos } & \multicolumn{2}{|c|}{ Ninfas } & \multicolumn{2}{|c|}{ Adultos } & \multicolumn{2}{|c|}{ Larvas } & \multicolumn{2}{|c|}{ Ninfas } & \multicolumn{2}{|c|}{ Adultos } & & \\
\hline & $\mathrm{N}$ & $\%$ & $\mathrm{~N}$ & $\%$ & $\mathrm{~N}$ & $\mathrm{~N}$ & $\%$ & $\%$ & $\mathrm{~N}$ & $\%$ & $\mathrm{~N}$ & $\%$ & $\mathrm{~N}$ & $\%$ & $\mathrm{~N}$ & $\%$ & $\mathrm{~N}$ & $\%$ \\
\hline Ave & 14 & 50 & 42 & 53,16 & 106 & 36,55 & 27 & 79,41 & 40 & 68,96 & 14 & 50,00 & 69 & 61,06 & 146 & 41,95 & 229 & 46,83 \\
\hline Roedor & 9 & 32,14 & 20 & 25,32 & 100 & 34,48 & 6 & 17,65 & 16 & 27,59 & 9 & 32,14 & 26 & 23,01 & 116 & 33,33 & 151 & 30,88 \\
\hline Gambá & 5 & 17,86 & 12 & 15,19 & 51 & 17,59 & 1 & 2,94 & 2 & 3,45 & 05 & 17,86 & 13 & 11,50 & 53 & 15,23 & 71 & 14,52 \\
\hline Bovino & 0 & 0 & 3 & 3,80 & 18 & 6,21 & 0 & 0 & 0 & 0 & 0 & 0 & 3 & 2,66 & 18 & 5,18 & 21 & 4,29 \\
\hline Equino & 0 & 0 & 2 & 2,53 & 15 & 5,17 & 0 & 0 & 0 & 0 & 0 & 0 & 2 & 1,77 & 15 & 4,31 & 17 & 3,48 \\
\hline Suino & 0 & 0 & 0 & 0 & 0 & 0 & 0 & 0 & 0 & 0 & 0 & 0 & 0 & 0 & 0 & 0 & 0 & 0 \\
\hline Total & 28 & 100 & 79 & 69,91 & 290 & 83,33 & 34 & 30,09 & 58 & 16,67 & 28 & 5,73 & 113 & 23,11 & 348 & 71,16 & 489 & 100 \\
\hline
\end{tabular}

As reações múltiplas ocorreram em 6,4\% (9/140) do total de ninfas e $20,2 \%(98 / 484)$ de adultos (Tab. 3). Sangue de aves ocorreu em $84,1 \%$ (90/107) dessas reações, enquanto, sangue de mamíferos foi detectado em 100\% (107/107) das amostras.

Tabela 3. Freqüências absoluta $(\mathrm{N})$ e relativa (\%) de hospedeiros detectados em reações multiplas no teste de precipitina, segundo o estádio de Argas (Persicargas) miniatus e o município pesquisado

\begin{tabular}{|c|c|c|c|c|c|c|c|c|c|c|c|c|c|c|c|c|c|c|}
\hline \multirow{3}{*}{ Hospedeiro } & \multicolumn{6}{|c|}{ Raphael Jambeiro - BA } & \multicolumn{4}{|c|}{ Gouveia - MG } & \multicolumn{6}{|c|}{ Total } & \multirow{2}{*}{\multicolumn{2}{|c|}{ Total geral }} \\
\hline & \multicolumn{2}{|c|}{ Larvas } & \multicolumn{2}{|c|}{ Ninfas } & \multicolumn{2}{|c|}{ Adultos } & \multicolumn{2}{|c|}{ Ninfas } & \multicolumn{2}{|c|}{ Adultos } & \multicolumn{2}{|c|}{ Larvas } & \multicolumn{2}{|c|}{ Ninfas } & \multicolumn{2}{|c|}{ Adultos } & & \\
\hline & $\mathrm{N}$ & $\%$ & $\mathrm{~N}$ & $\%$ & $\mathrm{~N}$ & $\%$ & $\mathrm{~N}$ & $\%$ & $\mathrm{~N}$ & $\%$ & $\mathrm{~N}$ & $\%$ & $\mathrm{~N}$ & $\%$ & $\mathrm{~N}$ & $\%$ & $\mathrm{~N}$ & $\%$ \\
\hline Ave/roedor & 0 & 0 & 1 & 20,00 & 32 & 36,36 & 4 & 100 & 10 & 100 & 0 & 0 & 5 & 55,56 & 42 & 42,86 & 47 & 43,92 \\
\hline Ave/gambá & 0 & 0 & 3 & 60,00 & 23 & 26,14 & 0 & 0 & 0 & 0 & 0 & 0 & 3 & 33,33 & 23 & 23,47 & 26 & 24,30 \\
\hline Ave/bovino & 0 & 0 & 0 & 0 & 9 & 10,23 & 0 & 0 & 0 & 0 & 0 & 0 & 0 & 0 & 9 & 9,18 & 9 & 8,41 \\
\hline Ave/eqüino & 0 & 0 & 0 & 0 & 4 & 4,54 & 0 & 0 & 0 & 0 & 0 & 0 & 0 & 0 & 4 & 4,08 & 4 & 3,74 \\
\hline Roedor/gambá & 0 & 0 & 0 & 0 & 9 & 10,23 & 0 & 0 & 0 & 0 & 0 & 0 & 0 & 0 & 9 & 9,18 & 9 & 8,41 \\
\hline Roedor/bovino & 0 & 0 & 0 & 0 & 4 & 4,54 & 0 & 0 & 0 & 0 & 0 & 0 & 0 & 0 & 4 & 4,08 & 4 & 3,74 \\
\hline Gambá/bovino & 0 & 0 & 1 & 20,0 & 3 & 3,41 & 0 & 0 & 0 & 0 & 0 & 0 & 1 & 11,11 & 3 & 3,06 & 4 & 3,74 \\
\hline Ave/roedor/gamá & 0 & 0 & 0 & 0 & 3 & 3,41 & 0 & 0 & 0 & 0 & 0 & 0 & 0 & 0 & 3 & 3,06 & 3 & 2,80 \\
\hline Ave/roedor/bovino & 0 & 0 & 0 & 0 & 1 & 1,14 & 0 & 0 & 0 & 0 & 0 & 0 & 0 & 0 & 1 & 1,02 & 1 & 0,94 \\
\hline Total & 0 & 0 & 5 & 55,56 & 88 & 89,80 & 4 & 44,44 & 10 & 10,20 & 0 & 0 & 9 & 8,41 & 98 & 91,59 & 107 & 10 \\
\hline
\end{tabular}

\section{DISCUSSÃO}

Hoogstraal e Kim (1985) consideram que modificações na especificidade de carrapatos em relação ao hospedeiro podem surgir quando hospedeiros fisiologicamente aceitáveis sejam introduzidos na relação primária parasitohospedeiros. Entretanto, Klompen et al. (1996) ao analisarem a teoria de que carrapatos tendem a ser hospedeiros específicos, concluíram que o nível de especificidade estaria associado à freqüência e ao ambiente de coleta de uma amostra. Nesse aspecto, A. miniatus tende a ser considerado bastante específico, uma vez que sua presença no hospedeiro é temporária, sendo freqüentemente localizado no ambiente onde este vive, em locais bem protegidos como ninhos, toco de árvores e poleiros, alimentando-se preferencialmente à noite (Flechtmann, 1973; Hoogstraal e Aeschlimann, 1982; Camicas et al., 1998; Serra-Freire, 2001). Assim, hospedeiros nidícolas e que tendem a formar colônias, como aves, são mais facilmente encontrados parasitados por esse carrapato. Entretanto, o encontro de $A$. miniatus em mamíferos tem sido raramente relatado (Doss et al., 1974), apesar de, experimentalmente, ter sido comprovada a conclusão de seu ciclo biológico quando alimentados com sangue em mamífero (Fonseca et al., 1999).

Mesmo considerando que a especificidade pode influenciar a adaptação parasitária, não são consideradas como relação de causa e efeito 
(Brooks e McLennan, 1993). Embora a adaptação a um hospedeiro possa direcionar a especificidade, esta não é, necessariamente, um indicador de adaptação. Assim, a especificidade pode surgir pela falta de oportunidade de transferência do parasito para hospedeiros alternativos, ou como efeito secundário da adaptação ao ambiente. (Klompen et al., 1995; 1996). Desse modo, a adaptação do A. miniatus a um habitat comum às aves permite o encontro rotineiro desse carrapato nestes hospedeiros, mas não exclui a possibilidade do parasitismo em outro táxon, como mamíferos, por exemplo.

Dessa forma, os resultados ora obtidos corroboram com essa hipótese ao apontarem para a polichevia e inespecificidade, que envolvem uma ampla variedade de hospedeiros de grupos filogenéticos distintos, em áreas fisiográficas distintas, mas que mantêm, como característica comum, o tipo de nicho onde os espécimes foram coletados. Igualmente, a freqüencia de indivíduos, nos diferentes estádios, que se alimentaram exclusivamente em mamíferos, ou conseguiram buscar várias fontes de alimentação sinaliza para o potencial de adaptação de $A$. miniatus, considerando-se a disponibilidade de fonte alimentar e a manutenção de seu ciclo de vida.

\section{REFERÊNCIAS BIBLIOGRÁFICAS}

ARAGÃO, H.; FONSECA, F. Notas de Ixodologia. VIII. Lista e chave para os representantes da fauna ixodológica brasileira. Mem. Inst. Oswaldo Cruz, v.59, p.115-129, 1961.

BROOKS, D.R.; McLENNAN, D.A. Parascript. Parasites and the language of evolution. Washington: Smithsonian Institute, 1993. 429p.

DOSS, M.A.; FARR M.M.; ROACH, K.F. et al. Ticks and tickborne diseases - I: Genera and species of ticks. Index catalogue of medical and veterinary zoology. Special publication n.3. Part 1. Washington: United States Department of Agriculture, 1974. 593p.

CAMICAS, J.L.; HERVY, J.P.; ADAM, F. et al. Les tiques $d u$ monde. Nomenclature, stades décrits, hôtes, répartition (Acárida, Ixodida). Paris: Éditions de l'Orstom, 1998. 233p.
EVANS, D.E.; MARTINS, J.R.; GUGLIELMONE, A.A. A review of the ticks (Acari, Ixodida) of Brazil, their hosts and geographic distribution - 1. The State of Rio Grande do Sul, Southern Brazil. Mem. Inst. Oswaldo Cruz, v.95, p.453-470, 2000.

FLECHTMANN, C. Ácaros de importância médica e veterinária. São Paulo: Nobel S.A., 1973. 191p.

FONSECA, A.H.; DUTRA, A.E.A.; PINA, I.G. Uso de sangue de frangos e de bovinos na alimentação de Argas (Persicargas) miniatus (Koch, 1848) (Acari, Argasidae), através de membrana de silicone. Rev. Bras. Cienc. Vet., v.6, p.167-170, 1999.

HOOGSTRAAL, H.; AESCHLIMANN, A. Tick-host specificity. Bull. Soc. Entomol. Suisse, v.55, p.5-32, 1982.

HOOGSTRAAL, H.; KIM, K.C. Ticks and mammal coevolution, with emphasis on Haemaphysalis: 505-568. In: KIM, K.C. (Ed.). Coevolution of parasitic arthropods and mammals. New York: Wiley \& Sons, 1985. 800 p.

KLOMPEN, J.S.H.; BLACK IV, W.C.; KEIRANS, J.E. et al. Evolution of ticks. Annu. Rev. Entomol., v.41, p.141-161, 1996.

KLOMPEN, J.S.H.; KEIRANS, J.E.; DURDEN, L.A. Three news species of ticks (Ixodida: Argasida: Carios) on a fruit bats (Chiroptera: Pteropodidae) in the Australasian region, with notes on host association. Acarologia, v.36, p.2540, 1995.

LOROSA, E.S.; ANDRADE, R.E.; SERRA, M.G.A. et al. Estudo de fontes alimentares através da técnica de precipiotina e grau de infectividade em Triatoma rubrofasciata (De Geer, 1773) coletados na Ilha de São LuísMaranhão. Entomol. Vect., v.5, p.241-250, 1998.

SERRA-FREIRE N.M. Ácaros (carrapatos e outros): In: MARCONDES, C.B. (Ed.). Entomologia médica veterinária. São Paulo: Atheneu, 2001. 432p.

SIQUEIRA, A.F. Estudos sobre a reação de precipitina aplicada à identificação de sangue ingerido por triatomíneos. Rev. Inst. Med. Trop. São Paulo, v.2, p.41-53, 1960. 